\title{
Temporal relationships between systemic lupus erythematosus and
}

\section{comorbidities}

Chang-Fu Kuo, MD PhD; ${ }^{1,2^{*}}$ I-Jun Chou, MD; ${ }^{3,4^{*}}$ Frances Rees, MD PhD; ${ }^{1}$ Matthew J Grainge, PhD; ${ }^{5}$ Peter Lanyon, DM FRCP MRCGP; ${ }^{1,6}$ Graham Davenport, MA MSc MB FRCGP; ${ }^{7}$ Christian Mallen, MD; ${ }^{8}$ Ting-Ting Chung, MSc; ${ }^{2}$ Jung-Sheng Chen, MSc; ${ }^{2}$ Weiya Zhang, $\mathrm{PhD}{ }^{1}$ Michael Doherty, $\mathrm{MD}^{1}$

\section{Author affiliations:}

1. Division of Rheumatology, Orthopaedics, and Dermatology, School of Medicine, University of Nottingham, Nottingham, UK; 2. Division of Rheumatology, Allergy and Immunology and Center for Artificial Intelligence in Medicine, Chang Gung Memorial Hospital, Taoyuan, Taiwan; 3.Division of Clinical Neuroscience, School of Medicine, University of Nottingham, Nottingham, UK; 4. Division of Paediatric Neurology, Chang Gung Memorial Hospital, Taoyuan, Taiwan; 5. Division of Epidemiology and Public Health, School of Medicine, University of Nottingham, Nottingham, UK; 6. Rheumatology Department, Nottingham University Hospitals NHS Trust, Nottingham, UK; 7. Arthritis Research UK Primary Care Centre, Keele University, Keele, UK; 8. Primary Care and Health Sciences, Keele University, UK.

Word count: 3541

Conflict of interest disclosures: None to declare

Joint senior authors: Drs Weiya Zhang and Michael Doherty

Correspondence to: Dr Changfu Kuo

Division of Rheumatology, Orthopaedics and Dermatology, School of Medicine, University of Nottingham, Nottingham, UK

Email: zandis@gmailc.com; Telephone: +44 (0) 1158231754 
Address: Academic Rheumatology, The University of Nottingham, Clinical Sciences Building, Nottingham City Hospital, Hucknall Road

Nottingham, NG5 1PB, UK 


\begin{abstract}
Objective To examine the burden of comorbidities prior to and after the diagnosis of systemic lupus erythematosus (SLE) and its impact on mortality.

Methods We identified 1605 incident cases of SLE and 6284 matched controls from the UK primary care. The risks of comorbidities before (prevalence; odds ratios (ORs)) and after SLE diagnosis (incidence; hazard ratios (HRs)) and the impact of comorbidities at diagnosis on allcause mortality were estimated.
\end{abstract}

Results At diagnosis SLE was associated with adjusted ORs (95\% confidence interval [CI]) of 2.25 (1.97-2.56), 3.37 (2.49-4.57) and 3.54 (1.89-6.63) for a Charlson comorbidity index of 12, 3-4 and $\geq 5$, respectively. Following diagnosis SLE also associated with increased risk of developing any comorbidity with an adjusted $\mathrm{HR}(95 \% \mathrm{Cl})$ of $1.30(95 \% \mathrm{Cl}, 1.13-1.49)$. At diagnosis SLE was associated with a greater risk of cancer, cardiovascular, renal, liver, rheumatological and neurological diseases as well as depression, anaemia and psoriasis. Risks of developing incident comorbidity in the categories of neoplasm, cardiovascular, genitourinary, metabolic/endocrine, gastrointestinal and hepatic diseases, chronic pulmonary diseases, musculoskeletal/connective tissue and neurological diseases were higher in SLE patients. People with SLE had higher mortality risk compared with controls, with adjusted $\mathrm{HR}$ of $1.91(95 \% \mathrm{Cl}, 1.62-2.26)$; after further adjusting for comorbidities this reduced to 1.64 (1.37-1.97). Comorbidities at SLE diagnosis accounted for $27.6 \%$ of the apparent difference in mortality between SLE patients and matched controls.

Conclusions People with SLE have increased risks of multiple comorbidities both prior to and after diagnosis and this contributes significantly to all-cause mortality. 


\section{Keywords}

1. Systemic lupus erythematosus

2. Comorbidities

3. Temporal relationships

4. All-cause mortality

5. Charlson comorbidity index

6. Epidemiology

\section{Key message:}

1. Patients with SLE have a higher burden of multiple comorbidities at and after diagnosis.

2. Comorbidities contribute significantly to all-cause mortality for SLE patients.

3. A thorough search for comorbidities and observation are warranted for SLE patients from diagnosis. 


\section{Introduction}

Systemic lupus erythematosus (SLE) is a female-predominant prototypic autoimmune disease characterised by autoantibody production and subsequent multiple target organ damage. $[1,2]$ SLE can occur at any age and the course of the disease is highly diverse and unpredictable.[3] SLE can involve any part of the body but specific organ damage to the heart, joints, skin, lungs, blood vessels, liver, kidneys, and nervous system define most clinical manifestations.[4] Both the multi-organ involvement of SLE and treatment for SLE frequently lead to an increased risk for comorbidities in affected patients[5] which collectively impacts adversely on the prognosis.[6] For example, people with SLE are reported to have an almost $80 \%$ higher mortality than the general population.[7] Current EULAR (European League against Rheumatism) recommendations for the management of SLE advise a high index of suspicion for the occurrence of comorbidities which should be promptly evaluated.[8] The ACR (American College of Rheumatology) guidelines for screening, treatment and management of lupus nephritis specifically recommend high vigilance for the control of hypertension and hypercholesterolemia.[9] However, other types of comorbidities may also associate with SLE. For example, important comorbidities including hypertension, dyslipidaemia, diabetes, osteoporosis, avascular necrosis, and malignancies are also more common in people with SLE.[10] A recent population-based study using the Clinical Practice Research Data-link (CPRD), which is broadly representative of the UK general population documented a higher risk for cardiovascular diseases, stroke, end-stage renal disease, cancer, osteoporosis, and infection in people with SLE.[5] However, existing evidence predominantly comes from tertiary referral centres, which may be biased towards more severe cases, and additionally suboptimal selection of appropriate 
controls hinders interpretation of the risks. Furthermore, how comorbidities at diagnosis impact on prognosis has not been examined. Therefore, we conducted this populationbased study using the CPRD to examine the risk of a comprehensive range of comorbidities in people with SLE compared with matched controls firstly at the time of the initial diagnosis of SLE and secondly during follow-up subsequent to diagnosis. We further examined the relative contribution of comorbidities to all-cause mortality.

\section{Methods}

This study used a hybrid design to combine both retrospective (prior to SLE diagnosis) and subsequent follow-up data (after initial diagnosis) from the CPRD. In addition, we modelled the influence of existing comorbidities on the all-cause mortality of SLE. The study was approved by the Independent Scientific Advisory Committee for MHRA Database Research (protocol 13_092).

\section{Data source}

We used the CPRD to examine the risk of comorbidities before and after the first diagnosis of SLE in incident patients identified between 1997 and 2005. The CPRD is an anonymised longitudinal database from the UK general practice containing individual level medical records, which was incepted in 1987 and includes approximately 14 million individuals in the UK up to 2017. In any calendar year covered, approximately $7 \%$ of the UK population were included in the database.[11] This database includes comprehensive information on demographics, lifestyle factors, medical diagnoses, results of investigations and examinations, operations, consultations, referrals and prescribed medications. The diagnostic coding system in CPRD is the Read code and the coding lists used in this study to 
identify people with SLE and an array of comorbidities are shown in supplements. The database has been well validated for many diagnoses.[12-16]

\section{Definition of systemic lupus erythematosus}

People with a new diagnosis of SLE from 1997 to 2005 who had no evidence of SLE prior to the time of diagnosis (index date) and had at least a 3-year continuous registration prior to the index date were identified. To ensure the data quality recorded by each general practitioner (GP), we only included individuals registered with up-to-standard practices. Read codes which we used to identify SLE are listed in supplementary table 1 . This code list was developed by our research team as described in our previous publications. $[5,7,17]$ We selected Read codes for SLE or a subtype of SLE excluding cutaneous only lupus (such as discoid lupus) from the CPRD medical dictionary browser. Codes were excluded if they represented a diagnostic test, a scoring system, tuberculosis, lupus pernio, cutaneous-only SLE, drug-induced lupus or neonatal lupus. These code lists were developed by 3 rheumatologists, 1 epidemiologist, 1 statistician and CPRD expert and 1 general practitioner. [17] We did not do external validation since GPs generally based on a confirmation by a hospital specialist and the positive predictive values of other autoimmune diseases on the CPRD were $>90 \% .[1-3]$

\section{Selection of controls}

The control group were participants from up-to-standard practices between 1997 and 2005 who had at least 3 years of continuous registration and no record of SLE prior to the index date. We matched SLE cases 1:4 to controls by year of birth ( \pm 2 years), gender, general practice and year of first continuous registration ( \pm 2 years). The index date was the initial diagnosis date of SLE in cases, with the same date applied to their matched controls. 


\section{Study period}

The comorbidity status of people with SLE was ascertained in each of three periods: (1) 10 years prior to index date; (2) 1 year prior to index date; and (3) after index date to the earliest date of occurrence of comorbidity, death, transfer from a participating general practice or 31 December 2013, whichever came first. The reason to include the two period prevalence (10 years and 1 year) prior to index date was to assess potential bias due to different observational periods for prevalence data.

\section{Comorbidity and mortality}

The general health status of both people with SLE and their matched controls was measured by the Charlson comorbidity index,[18] which contains 17 categories of comorbidities (myocardial infarction, congestive heart failure, peripheral vascular disease, cerebrovascular disease, dementia, chronic pulmonary disease, rheumatological disease (excluding SLE), peptic ulcer disease, mild liver disease, moderate or severe liver disease, diabetes mellitus (DM), DM with chronic complications, renal diseases, any malignancy (including leukaemia and lymphoma), metastatic solid tumour and HIV infection. We calculated the Charlson score for each study person at baseline by adding scores assigned to each specific diagnosis. Those coding lists were validated by Deyo et al. who produced a version for use with the International Classification of Diseases, Ninth Revision (ICD-9) based database,[19] which was the basis of the validated Read code version.[20]

In addition, we also incorporated other comorbidities potentially associated with SLE, including anaemia, cardiac arrhythmias, depression, hypertension, hypothyroidism, gout, multiple sclerosis, neurological diseases, osteoarthritis, psoriasis, psychosis, urolithiasis and valvular heart disease. All comorbidities were further grouped into eight categories. The 
case definitions of these conditions were based on physician diagnoses recorded as Read codes. These comorbidities were identified separately from records within each of the three study periods. The Charlson index at diagnosis was based on results from the 10 -year period. Charlson comorbidity index was classified into categories: (1) 0, 1-2, 3-4 and $\geq 5$ scores; and (2) dichotomised as 0 and $\geq 1$ score. The code lists for these comorbidites are shown in supplementary table 2 .

Mortality was identified on the recording in the main database. The CPRD has developed an algorithm to identify death and date of death for deceased participants. We previously conducted a validation study comparing the mortality recorded in the CPRD main database and that in the death registry provided by the Office of National Statistics (ONS).[21] The positive and negative predictive value of a CPRD recorded death were 0.93 and 1.00 , respectively. The difference between dates of death recorded in CPRD and ONS was less than 3 months in $98.5 \%$ of deceased patients.

\section{Statistical analysis}

We estimated the prevalence of a specific comorbidity by dividing the number of people ever diagnosed with a given comorbidity during the past 10-year period or 1-year period before the index date by the number of people with SLE or matched controls as denominators. Odds ratios (ORs) and 95\% confidence intervals ( $\mathrm{Cls}$ ) were used to estimate the association between SLE and each coexisting medical condition. Conditional logistic regression was used to estimate ORs and $95 \%$ Cls considering covariates including body mass index (BMI) category, smoking status and alcohol consumption. Missing data were coded as 'unknown'. Kaplan-Meier plots were used to estimate the cumulative probability of each comorbidity and all-cause mortality after the index date in people with SLE and those 
without. Since some people may have comorbidities prior to SLE diagnosis, we only included people who were at risk for a given comorbidity, i.e., not having such comorbidity at index date, to estimate HRs of a specific comorbidity. Cox proportional hazards models were used to estimate $\mathrm{HRs}$ and $95 \% \mathrm{Cls}$ were calculated for a Charlson index of one or more score, for each separate comorbidity and all-cause mortality. The HRs were adjusted by age, gender, index year, BMI categories, smoking status and alcohol consumption. We constructed three Cox proportional hazards models to examine HR for death associated with the diagnosis of SLE: model 1, unadjusted; model 2, adjusted for age, sex, index year, BMI class, smoking status and alcohol consumption; and model 3, adjusted for covariates in model 2 and all comorbidities which were already in existence at the time of the index date.

We further examined the relative contribution of existing comorbidities at SLE diagnosis to the all-cause mortality using the concept of relative attenuation of rate ratios that was developed by Birkmeyer et al,[22] involving assessment of the relation between the SLE and all-cause mortality first, and then estimating the contribution by excluding and then including comorbidities at diagnosis. The relative contribution of existing comorbidities was computed as [HRa-HRc] $\div[\mathrm{HRa}-1]$, where $\mathrm{HRa}$ is the $\mathrm{HR}$ for all-cause mortality which was adjusted for all aforementioned covariates without consideration of pre-existing comorbidities and HRc is HR further adjusted for the full array of comorbidities at diagnosis of SLE. All statistical analyses were performed using SAS statistical software, V.9.4.

\section{Results}

During 1997 to 2005 we identified 1605 incident cases of SLE. Their mean age was $50.8 \pm$ 16.2 years and $1311(81.7 \%)$ were women. The median observation periods (interquartile 
range) before and after index date were $14(8-23)$ and 9 (5-11) years respectively. They were age- and sex-matched to 6284 controls with the same age and sex structure (figure 1 for flow chart). People with SLE tended to have a lower BMI and a higher prevalence of smoking. Patient characteristics at diagnosis are shown in Table 1.

At index date the prevalence of people with a Charlson index $\geq 1$ was significantly higher in people with SLE (38.07\%) than in controls $(20.35 \% ; p<0.001)$. In an adjusted logistic regression model focussing on diagnoses in the 10-years prior to the index date, ORs (95\% Cls) were 2.25 (1.97-2.56), 3.37 (2.49-4.57) and 3.54 (1.89-6.63) for Charlson scores 1-2, 34 and $\geq 5$ respectively ( $p$ for trend $<0.001$ ). The analysis restricted to diagnoses recorded in the one-year period prior to the index date provided a similar pattern of associations (supplementary table 2). After SLE diagnosis, the median time to first comorbidity in the Charlson index was 28 months ( $95 \% \mathrm{Cl}, 21-35$ months) from the index date in people with SLE and 83 months ( $95 \% \mathrm{Cl}, 79-87$ months) in controls (log-rank test $p<0.001)$. The cumulative probability of having the first comorbidity in the Charlson index at 1, 2, 5 and 10 years from index date were $42.4 \%, 45.0 \%, 51.8 \%$ and $61.7 \%$ in people with SLE and $22.4 \%$, $24.2 \%, 30.0 \%$ and $39.6 \%$ in controls (supplementary table 3 ). In addition, the cumulative probability of any increase in Charlson score was significantly greater in people with SLE than in controls and SLE was associated with an adjusted HR (95\% Cl) of $1.80(1.62-2.26)$ for having a Charlson index $\geq 1$.

Renal diseases were particularly more common in people with SLE than matched controls at diagnosis, with an OR $(95 \% \mathrm{Cl})$ of 8.69 (5.21-14.50). Comparing to the estimates for comorbidities occurring in the period one year prior to SLE diagnosis (supplementary table 2), SLE was associated with an OR $(95 \% \mathrm{Cl})$ of 19.57 (9.08-42.2) for renal diseases, suggesting 
renal diseases often occur close to the time of SLE diagnosis. However, 9 out of 49 SLE patients who had existing renal diseases at diagnosis had kidney injury long before SLE diagnosis. After SLE diagnosis, the cumulative probability of having renal diseases were 4.39 at 1 year and 6.78 at 5 years, which corresponded to a $\mathrm{HR}(95 \% \mathrm{Cl})$ of $3.43(2.38-4.93)$ when compared to controls.

Other rheumatological diseases were common in SLE patient at diagnosis. When examining 10 years prior to SLE diagnosis, 211 out of 1605 SLE patients (13.15\%) had another rheumatological diagnosis (OR, 3.94). Restricting to one year prior to SLE diagnosis, there were still 108 (6.73\%) patients who were given another rheumatological diagnosis (OR, 4.73). After SLE diagnosis, there were 155 patients (11.12\%) of at-risk patients who were given a new diagnosis of another rheumatological disease, the $\mathrm{HR}(95 \% \mathrm{Cl})$ being $3.26(2.45-$ 4.33) when compared to controls. Overall, the cumulative probability of having another rheumatological diagnosis was 15.60 at 1 year and 19.60 at 5 years after SLE diagnosis. The risk of other comorbidities at and after diagnosis in SLE patients comparing to controls are summarized in Table 2, supplementary table 3 and 4.

After the index date, mortality at 5-years and 10 -years from any cause was $8.28 \%$ and $17.00 \%$ in people with SLE, compared to $4.43 \%$ and $10.29 \%$ in matched controls (log-rank test, $p<0.001$; supplementary table 5). Estimates of the effect of a diagnosis of SLE on mortality were attenuated after adjustment for demographic and lifestyle variables as well as comorbidity status at index date (table 3). After adjustment for age, sex, index year, BMI class, smoking status and alcohol consumption, SLE was associated with a HR of 1.91 (95\% $\mathrm{Cl}, 1.62-2.26)$. However, after adjustment for multiple comorbidities at diagnosis SLE remained strongly associated with all-cause mortality with a $\mathrm{HR}(95 \% \mathrm{Cl})$ of 1.64 (1.37-1.97). 
Thus, comorbidities at diagnosis of SLE accounted for $27.6 \%$ of the apparent difference in mortality between SLE patients and matched controls. The proportion of the apparent effect on mortality that was actually attributable to comorbidities at diagnosis was lower in men (20.6\%) than in women (30.7\%).

\section{Discussion}

This nationwide population-based study found an increased risk of comorbidity in people with SLE compared to matched controls at the time of initial diagnosis as well as an increased risk of incident comorbidity following diagnosis. Approximately half of people with SLE developed a condition included in the Charlson comorbidity index within 3 years of initial diagnosis, whereas it took 7 years for half of the controls to develop a relevant comorbidity. The pronounced differences in risks of an array of comorbidities between people with SLE prior to their initial diagnosis and matched controls suggest that a significant degree of organ damage and systemic consequences from SLE occur prior to initial diagnosis. This might be the reason for our finding that comorbidities at lupus diagnosis contributed $30 \%$ to all-cause mortality. Overall, the burden of comorbidity is substantially higher than controls at diagnosis of SLE and the risk of developing new comorbidity following diagnosis is also higher in people with SLE than in the general population. Furthermore, comorbidities contributed significantly to mortality. Therefore, a systematic screen for a range of comorbidities appears warranted in patients with SLE both at first diagnosis and subsequently during follow-up.

Previous studies have reported an increased risk of multiple comorbidities in people with SLE but the temporal relationships with diagnosis of SLE are reported rarely. Our previous 
study using CPRD data also found a greater comorbidity burden in prevalent SLE patients than controls, particularly for cardiovascular disease, stroke, renal failure, cancer, osteoporosis, and infection.[5] Other studies in general concur that the increased risk of comorbidities in selected categories, for example, cardiovascular and cerebrovascular diseases are consistently more common in people with SLE, regardless of age.[23-26] Chronic kidney disease is common in people with SLE and often leads to end-stage renal disease.[27, 28] Liver diseases, defined by biochemical or histological abnormalities, also seem more prevalent in people with SLE than controls.[29] The risk for cancer is also increased in people with SLE and a strong association between specific malignancies such as lung cancer, lymphoma, and other hematologic malignancies has been reported consistently.[30-32] People with SLE appear to be at greater risk of osteoporosis and subsequent fragility fractures compared to appropriate controls.[33, 34] Other categories of comorbidities that have been reported previously with established SLE include dyslipidaemia,[35] diabetes mellitus,[35] preclinical atherosclerosis,[36] and avascular bone necrosis.[37] The present study ascertained the risk of these comorbidities, as well as many other categories. Importantly we also identified the temporal course of the risk of comorbidities relative to the initial diagnosis of SLE.

The higher comorbidity burden found both at 1 year and 10 years prior to diagnosis suggests that accrual of comorbidity is already greater in people with SLE than controls prior to the initial diagnosis. Several reasons may explain such a phenomenon. Both a pro-inflammatory status and immune aberration have been found to exist even before the clinical presentation of SLE. For example, subclinical atherosclerosis may underlie the risk of myocardial infarction close to or before SLE diagnosis. Delayed diagnosis may partly explain the higher risk of 
certain comorbidities more closely linked to SLE, such as renal disease and CKD. However, the magnitude of odds ratios for specific comorbidities prior to diagnosis suggests that clinically occult specific organ damage may have occurred commonly prior to diagnosis. In addition, the accumulation of new comorbidities appears to be faster in people with SLE after diagnosis in both general and specific comorbidity categories. Therefore, at initial diagnosis of SLE, a comprehensive assessment of a wide range of comorbidities appears to be important, since some of these, such as osteoporosis and mild liver injury, present insidiously and their identification may influence management decisions. The continuous monitoring of the development of comorbidities also seems justified as part of standard care for people with SLE.

A higher mortality risk in patient with lupus has long been recognised. A recent study using US national database and census data between 1968 and 2013 found a significant improvement in all-cause mortality but the reduction in mortality attributed to SLE was less than non-SLE causes.[38] A recent meta-analysis comprising 27,123 patients with SLE (among them 4,993 deaths) reported an 3-fold higher mortality risk in SLE patients compared with the general population, specific causes of death mainly being cardiovascular diseases, malignancy, infection and renal diseases.[39] It seems reasonable that comorbidities may impact on the mortality, in addition to SLE itself, however, the actual contribution has not been assessed before. In this study, we found that the comorbidities at diagnosis are a genuine risk factor for mortality, contributing approximately $30 \%$ to the risk of death. Since we also observed an accelerated incidence of comorbidities in SLE patients, their existence impacts significantly on the management and care of SLE patients, both at diagnosis and during follow-up. 
The mechanisms underlying the relationships between SLE and the various associated comorbidities are complex and it is often difficult to discern causality, including from drugs (e.g. corticosteroids, immunosuppresants, NSAIDs) used to treat SLE following diagnosis .[40] Clinical implications of a greater comorbidity burden are simpler. The identification of comorbidity can be part of the diagnostic workup, as a consideration for management decisions and as a flag for prognosis.[41] Comorbidities such as CKD and anaemia are part of the classification criteria of SLE and awareness of the relationships in primary care between SLE and these closely related comorbidities may simplify the diagnosis of SLE. Using comorbidity as an indicator of prognosis is used widely in clinical practice. For example, the Charlson comorbidity index is used frequently to estimate comorbidity burden and to predict mortality.[18] Furthermore the presence of comorbidity may influence the management of SLE. Currently, EULAR recommendations for management of SLE advise a high index of suspicion and diligent follow-up for comorbidities in people with SLE.[42] The ACR guidelines for lupus nephritis also advise that special attention should be paid to possible presence of hypertension, hypercholesterolemia and vascular disease in people with SLE and that these should be treated appropriately.[9] However, our data indicate that the risks of comorbidities are not limited to cardiovascular or metabolic disease but involve multiple systems even at the time of initial diagnosis. Therefore, we suggest that a comprehensive comorbidity 'checklist', including all those that are more frequently present in people with SLE in this study, is justified to identify and treat comorbidities early and effectively to potentially improve patient outcomes.

There are several limitations to this study. Firstly, there may be some misclassification bias since the identification of people with SLE was based on physician diagnosis, rather than 
according to classification criteria. Similarly, there may have been some misclassification of comorbidities. However, many of these comorbidities are not typically diagnosed by general practitioners and such entries are likely to be guided by secondary care communication. Furthermore the diagnoses recorded in the CPRD have been validated previously with sufficient accuracy to support their use in clinical and epidemiological studies.[12] Although SLE has not been fully validated within the CPRD, validation of other autoimmune diseases within the CPRD has shown a good positive predictive value of over $90 \%$.[43, 44] In addition, there is no reason to suspect a differential misclassification. Secondly, differential ascertainment bias between people with SLE and controls cannot be excluded, even though the estimates based on different lengths of previous observation produced similar results. Thirdly, residual confounding may exist. Fourthly, SLE manifestations may be misclassified as a disease entity. For example, multiple sclerosis as a comorbidity may be a misclassification of neuropsychiatric lupus. Fifthly, completeness of data across patients and across time is variable and the patterns or causes of missing are complex.[11] These missing data, such as alcohol consumption, may also have impact on the interpretation of our results. Race is also not available for all subjects in the CPRD. Only $27.1 \%$ of all patients in the CPRD have ethnicity recorded and among them $86 \%$ were white British.[45]

In conclusion, a large proportion of people with SLE in the UK already have a greater than expected prevalence of comorbidity at the time of diagnosis and the subsequent risk of incident comorbidity following diagnosis is higher than in matched general population controls. All-cause mortality was higher in people with SLE and the increased risk may be caused both by SLE and its pre-existing or subsequent comorbidities. This study suggests that a thorough search for a broad range of comorbidity and subsequent vigilant observation 
should be considered for all people with SLE from the date of first diagnosis.

Funding: This work was funded by the National Science Council of Taiwan (project 104-2314B-182A-047) and Chang Gung Memorial Hospital (project CMRPG3E1961) and was supported by the University of Nottingham in methodology and infrastructure. CDM is funded by the NIHR Collaborations for Leadership in Applied Health Research and Care West Midlands, the NIHR School for Primary Care Research and an NIHR Research Professorship (NIHR-RP-2014-04-026). 


\section{Reference}

1 Petri M. Sex hormones and systemic lupus erythematosus. Lupus 2008;17:412-5.

2 Burch PR, Rowell NR. Lupus erythematosus. Analysis of the sex- and age-distributions of the discoid and systemic forms of the disease in different countries. Acta Derm Venereol 1970;50:293-301.

3 Alarcon GS, Roseman J, Bartolucci AA, et al. Systemic lupus erythematosus in three ethnic groups: II. Features predictive of disease activity early in its course. LUMINA Study Group. Lupus in minority populations, nature versus nurture. Arthritis Rheum 1998;41:117380.

4 Wallace DJ. Lupus : the essential clinician's guide. Oxford ; New York: Oxford University Press; 2008.

5 Rees F, Doherty M, Grainge M, Lanyon P, Davenport G, Zhang W. Burden of Comorbidity in Systemic Lupus Erythematosus in the UK, 1999-2012. Arthritis Care Res (Hoboken) 2016;68:819-27.

6 Ward MM, Pajevic S, Dreyfuss J, Malley JD. Short-term prediction of mortality in patients with systemic lupus erythematosus: classification of outcomes using random forests. Arthritis Rheum 2006;55:74-80.

7 Rees F, Doherty M, Grainge MJ, Lanyon P, Davenport G, Zhang W. Mortality in systemic lupus erythematosus in the United Kingdom 1999-2012. Rheumatology (Oxford) 2016;55:854-60.

8 Bertsias G, Gordon C, Boumpas DT. Clinical trials in systemic lupus erythematosus (SLE): lessons from the past as we proceed to the future--the EULAR recommendations for the management of SLE and the use of end-points in clinical trials. Lupus 2008;17:437-42.

9 Hahn BH, McMahon MA, Wilkinson A, et al. American College of Rheumatology guidelines for screening, treatment, and management of lupus nephritis. Arthritis Care Res (Hoboken) 2012;64:797-808.

10 Walsh SJ, Algert C, Rothfield NF. Racial aspects of comorbidity in systemic lupus erythematosus. Arthritis Care Res 1996;9:509-16.

11 Herrett E, Gallagher AM, Bhaskaran K, et al. Data Resource Profile: Clinical Practice Research Datalink (CPRD). Int J Epidemiol 2015;44:827-36.

12 Herrett E, Thomas SL, Schoonen WM, Smeeth L, Hall AJ. Validation and validity of diagnoses in the General Practice Research Database: a systematic review. Br J Clin Pharmacol 2010;69:4-14.

13 Jick $\mathrm{H}$, Jick SS, Derby LE. Validation of information recorded on general practitioner based computerised data resource in the United Kingdom. BMJ 1991;302:766-8.

14 Khan NF, Harrison SE, Rose PW. Validity of diagnostic coding within the General Practice Research Database: a systematic review. Br J Gen Pract 2010;60:e128-36.

15 Jick SS, Kaye JA, Vasilakis-Scaramozza C, et al. Validity of the general practice research database. Pharmacotherapy 2003;23:686-9.

16 Meier $\mathrm{CR}$, Jick H. Omeprazole, other antiulcer drugs and newly diagnosed gout. Br J Clin Pharmacol 1997;44:175-8.

17 Rees F, Doherty M, Grainge M, Davenport G, Lanyon P, Zhang W. The incidence and prevalence of systemic lupus erythematosus in the UK, 1999-2012. Ann Rheum Dis 2016;75:136-41.

18 Charlson M, Szatrowski TP, Peterson J, Gold J. Validation of a combined comorbidity 
index. J Clin Epidemiol 1994;47:1245-51.

19 Deyo RA, Cherkin DC, Ciol MA. Adapting a clinical comorbidity index for use with ICD-9CM administrative databases. J Clin Epidemiol 1992;45:613-9.

20 Khan NF, Perera R, Harper S, Rose PW. Adaptation and validation of the Charlson Index for Read/OXMIS coded databases. BMC Fam Pract 2010;11:1.

21 Kuo CF, Grainge MJ, Mallen C, Zhang W, Doherty M. Comorbidities in patients with gout prior to and following diagnosis: case-control study. Ann Rheum Dis 2016;75:210-7.

22 Birkmeyer JD, Stukel TA, Siewers AE, Goodney PP, Wennberg DE, Lucas FL. Surgeon volume and operative mortality in the United States. N Engl J Med 2003;349:2117-27.

23 Moss KE, Ioannou Y, Sultan SM, Haq I, Isenberg DA. Outcome of a cohort of 300 patients with systemic lupus erythematosus attending a dedicated clinic for over two decades. Ann Rheum Dis 2002;61:409-13.

24 Fischer LM, Schlienger RG, Matter C, Jick H, Meier CR. Effect of rheumatoid arthritis or systemic lupus erythematosus on the risk of first-time acute myocardial infarction. Am J Cardiol 2004;93:198-200.

25 Ward MM. Premature morbidity from cardiovascular and cerebrovascular diseases in women with systemic lupus erythematosus. Arthritis Rheum 1999;42:338-46.

26 Mok CC, Ho LY, To CH. Annual incidence and standardized incidence ratio of cerebrovascular accidents in patients with systemic lupus erythematosus. Scand J Rheumatol 2009;38:362-8.

27 Mok CC, Tang SS. Incidence and predictors of renal disease in Chinese patients with systemic lupus erythematosus. Am J Med 2004;117:791-5.

$28 \mathrm{Yu} \mathrm{KH,} \mathrm{Kuo} \mathrm{CF,} \mathrm{Chou} \mathrm{IJ,} \mathrm{Chiou} \mathrm{MJ,} \mathrm{See} \mathrm{LC.} \mathrm{Risk} \mathrm{of} \mathrm{end-stage} \mathrm{renal} \mathrm{disease} \mathrm{in} \mathrm{systemic}$ lupus erythematosus patients: a nationwide population-based study. Int J Rheum Dis 2016;19:1175-82.

29 Bessone F, Poles N, Roma MG. Challenge of liver disease in systemic lupus erythematosus: Clues for diagnosis and hints for pathogenesis. World J Hepatol 2014;6:394409.

30 Dey D, Kenu E, Isenberg DA. Cancer complicating systemic lupus erythematosus--a dichotomy emerging from a nested case-control study. Lupus 2013;22:919-27.

31 Bernatsky S, Ramsey-Goldman R, Labrecque J, et al. Cancer risk in systemic lupus: an updated international multi-centre cohort study. J Autoimmun 2013;42:130-5.

32 Sultan SM, Ioannou Y, Isenberg DA. Is there an association of malignancy with systemic lupus erythematosus? An analysis of 276 patients under long-term review. Rheumatology (Oxford) 2000;39:1147-52.

33 Ramsey-Goldman R, Dunn JE, Huang CF, et al. Frequency of fractures in women with systemic lupus erythematosus: comparison with United States population data. Arthritis Rheum 1999;42:882-90.

34 Yee CS, Crabtree N, Skan J, et al. Prevalence and predictors of fragility fractures in systemic lupus erythematosus. Ann Rheum Dis 2005;64:111-3.

35 Bruce IN, Urowitz MB, Gladman DD, Ibanez D, Steiner G. Risk factors for coronary heart disease in women with systemic lupus erythematosus: the Toronto Risk Factor Study.

Arthritis Rheum 2003;48:3159-67.

36 Jimenez S, Garcia-Criado MA, Tassies D, et al. Preclinical vascular disease in systemic lupus erythematosus and primary antiphospholipid syndrome. Rheumatology (Oxford) 2005;44:756-61.

37 Gladman DD, Urowitz MB, Chaudhry-Ahluwalia V, Hallet DC, Cook RJ. Predictive factors 
for symptomatic osteonecrosis in patients with systemic lupus erythematosus. J Rheumatol 2001;28:761-5.

38 Yen EY, Shaheen M, Woo JMP, et al. 46-Year Trends in Systemic Lupus Erythematosus Mortality in the United States, 1968 to 2013: A Nationwide Population-Based Study. Ann Intern Med 2017;167:777-85.

39 Yurkovich M, Vostretsova K, Chen W, Avina-Zubieta JA. Overall and cause-specific mortality in patients with systemic lupus erythematosus: a meta-analysis of observational studies. Arthritis Care Res (Hoboken) 2014;66:608-16.

40 Rhee SH, Hewitt JK, Lessem JM, Stallings MC, Corley RP, Neale MC. The validity of the Neale and Kendler model-fitting approach in examining the etiology of comorbidity. Behav Genet 2004;34:251-65.

41 Feinstein AR. The Pre-Therapeutic Classification of Co-Morbidity in Chronic Disease. J Chronic Dis 1970;23:455-68.

42 Bertsias G, loannidis JP, Boletis J, et al. EULAR recommendations for the management of systemic lupus erythematosus. Report of a Task Force of the EULAR Standing Committee for International Clinical Studies Including Therapeutics. Ann Rheum Dis 2008;67:195-205.

43 Schoonen WM, Kucera G, Coalson J, et al. Epidemiology of immune thrombocytopenic purpura in the General Practice Research Database. Br J Haematol 2009;145:235-44.

44 Watts RA, Al-Taiar A, Scott DG, Macgregor AJ. Prevalence and incidence of Wegener's granulomatosis in the UK general practice research database. Arthritis Rheum 2009;61:14126.

45 Mathur R, Bhaskaran K, Chaturvedi N, et al. Completeness and usability of ethnicity data in UK-based primary care and hospital databases. J Public Health (Oxf) 2014;36:684-92. 